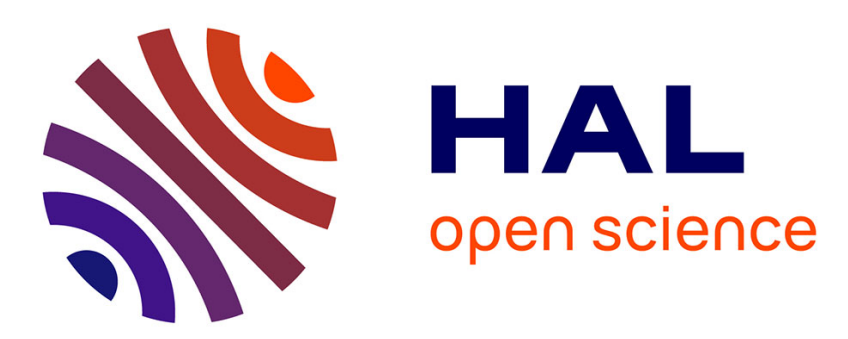

\title{
Influence de la température sur la courbe de rétention d'eau de milieux poreux
}

\author{
Simon Salager, Frédéric Jamin, Moulay Saïd El Youssoufi, Christian Saix
}

\section{To cite this version:}

Simon Salager, Frédéric Jamin, Moulay Saïd El Youssoufi, Christian Saix. Influence de la température sur la courbe de rétention d'eau de milieux poreux. Comptes Rendus Mécanique, 2006, 334 (6), pp.393 - 398. 10.1016/j.crme.2006.05.003 . hal-01412547

\section{HAL Id: hal-01412547 https://hal.science/hal-01412547}

Submitted on 8 Dec 2016

HAL is a multi-disciplinary open access archive for the deposit and dissemination of scientific research documents, whether they are published or not. The documents may come from teaching and research institutions in France or abroad, or from public or private research centers.
L'archive ouverte pluridisciplinaire HAL, est destinée au dépôt et à la diffusion de documents scientifiques de niveau recherche, publiés ou non, émanant des établissements d'enseignement et de recherche français ou étrangers, des laboratoires publics ou privés. 


\title{
Influence de la température sur la courbe de rétention d'eau de milieux poreux
}

\author{
Simon Salager ${ }^{\mathrm{a}, *}$, Fredéric Jamin ${ }^{\mathrm{b}}$, Moulay Saïd El Youssoufi a \\ Christian Saix ${ }^{\text {a }}$ \\ a Laboratoire de mécanique et génie civil, UMR 5508 CNRS-Université Montpellier II, CC048, place Eugène-Bataillon, \\ 34095 Montpellier cedex 05, France \\ b Bureau ALPES CONTROLES SA, ZA le Puech Radier, Bâtiment 7, rue Montels l'Eglise, 34970 Lattes, France
}

\section{Résumé}

Cette Note concerne l'influence de la température sur la courbe de rétention d'eau d'un milieu poreux. On présente un modèle, basé sur la différentielle de la succion en fonction de la température, de la teneur en eau et de l'indice des vides. Connaissant la courbe de rétention à une température donnée, le modèle permet de la prédire pour d'autres températures. Des résultats d'essais expérimentaux, réalisés à 20 et $60^{\circ} \mathrm{C}$, sur une céramique (terre cuite) et un sable limoneux-argileux, permettent de valider le modèle. L'application du modèle à d'autres résultats de la littérature montre sa capacité à couvrir une large gamme de matériaux poreux.

Influence of temperature on the water retention curve of porous media. This paper concerns the influence of temperature on the water retention curve of porous media. We present a model based on the differential of suction as a function of temperature, water content and void ratio. When adjusted for a given temperature, this model is able to predict the curve for any temperature. The model was validated by several tests on a ceramic (terra cotta) and a clayey silty sand at 20 and $60{ }^{\circ} \mathrm{C}$. The application of the model to data found in the literature confirms its predictive power for a wide range of porous materials.

Mots-clés : Milieux poreux; Sols ; Courbe de rétention ; Modèle thermo-hydrique

Keywords: Porous media; Soils; Water retention curve; Thermo-hydric model

* Auteur correspondant.

Adresses e-mail : salager@lmgc.univ-montp2.fr (S. Salager), fjamin@alpes-controles.fr (F. Jamin), elyous@1mgc.univ-montp2.fr

(M.S. El Youssoufi), saix@1mgc.univ-montp2.fr (C. Saix). 


\section{Abridged English version}

The water retention curve is the constitutive relation between gravimetric water content $w$ (or volumetric water content $\theta$ ) and suction $s$, for a given temperature $T$ and at a given void ratio $e$. It depends on the porosity, grainsize distribution, pore-geometry characteristics, and the mineralogical type of the solid phase. This paper presents a contribution to the characterization of the thermo-hydric behaviour of unsaturated porous media. A theoretical model allows us to predict the water retention curve for any temperature from a curve obtained experimentally at a given temperature. The model takes into account the thermal expansion of different phases and the surface tension of pore water and contact angle variations. It is based on the development of the differential of suction with respect to the volumetric water content, temperature and void ratio. To avoid the hysteresis phenomena, we consider here only drainage path from a saturated state.

The suction differential is expressed by (1). Relation (2) gives the volumetric water content as a function of the densities of solid and liquid phases $\left(\rho_{s}^{*}\right.$ and $\rho_{e}^{*}$ ), the gravimetric water content, and the void ratio. Then we express (relation (3)) the differential $\mathrm{d} \theta$, by introducing the volumetric thermal expansion coefficients of water $\beta_{e}$ and of the solid phase $\beta_{s}$. Eq. (2) also leads to (4). We consider here the funicular state for which the liquid phase is continuous in the porous media and transfers being made by liquid flows. Water is in a capillary state in the pores, so we can use the Jurin's law (5): the suction is expressed as a function of the surface tension of water $\sigma_{s}$, the mean radius of pores $r$, and the contact angle $\phi$. From (5), we determine the expression (6) which gives the derivative of suction with respect to temperature. $F_{w}$ is the reciprocal slope of the retention curve for a given temperature $\left(F_{w}=\left(\frac{\partial s}{\partial w}\right)_{T, e}\right)$. Introducing (3), (4), and (6) in Eq. (1), we obtain the final form (7) of the differential of suction. The variation of the contact angle versus temperature is very difficult to measure. One can note few results in literature about this subject. Thus, in our experimental validation of the model, we will not take this variation into account. Furthermore, the volumetric thermal expansion coefficient of the solid phase is assumed to be negligible, compared with volumetric thermal expansion coefficient of water. Under these assumptions, relation (7) can be written in the simplified form (8). Finally, from a retention curve obtained at the temperature $T_{0}$, the relation (8) allows to predict, for each suction value, the water content variation as a function of the temperature variation.

We used a pressure cell (Fig. 1). Suction is applied using the method of air over-pressure. The sample, put on a ceramic plate with a high air-entry pressure, is placed in an airtight cell where the air pressure is regulated by a pneumatic system. The airtight cell is immersed in a thermostatic bath. This device allows us to obtain retention curves for a porous media at several temperatures. The experiments were performed on two materials: a ceramic and a clayey silty sand. The experimental retention curves of the ceramic (Fig. 2(a)) and the clayey silty sand (Fig. 2(b)) at 20 and $60^{\circ} \mathrm{C}$ are modelled from experimental points using the fitting function of Fredlund and Xing [11]. From the retention curves at $20^{\circ} \mathrm{C}$, the model enables us to obtain this curves at $60^{\circ} \mathrm{C}$. The experiments and modelling are in good agreement for the ceramic. In the case of the clayey silty sand, one can note a divergence after a suction which corresponds to the transition between the funicular and the pendular state.

To complete the validation, we applied our model to data found in the literature on two different clays. The first is a FoCa compacted clay [7] and the second is prepared from Boom clay [8]. Fig. 3(a) shows the results obtained for the FoCa compacted clay; the temperatures of the tests were 20 and $80^{\circ} \mathrm{C}$. Experimental results at $80^{\circ} \mathrm{C}$ are in good agreement with the modelling results. Fig. 3(b) gives Romero's results [8] on the Boom clay. These tests were made for two different temperatures $\left(22\right.$ and $\left.80^{\circ} \mathrm{C}\right)$ and for a dry unit weight $\left(16.7 \mathrm{kN} \mathrm{m}^{-3}\right)$. In this case, our model is still in good agreement with experimental data. Other results obtained by Romero [8], in the same conditions but with a dry unit weight $\left(13.7 \mathrm{kN} \mathrm{m}^{-3}\right)$, were well predicted by the model. Finally, these results allow us to plot the water retention surface, obtained from experimental tests at a given temperature and from modelling. It can be considered as a generalization of the classical water retention curve.

\section{Introduction}

La caractérisation du comportement hydrique d'un milieu poreux est souvent présentée en terme de courbe de rétention d'eau qui exprime, pour une température $T$ donnée, la teneur en eau volumique $\theta$ (ou massique $w$ ), en fonction de la succion $s$. L'allure de cette courbe dépend en particulier de la porosité, de la granulométrie, de la tortuosité du réseau poreux et de la nature minéralogique de la phase solide. Au delà de la caractérisation du comportement 
hydrique, cette courbe a pu être utilisée pour la détermination du coefficient relatif de perméabilité [1,2] ou encore pour l'analyse de la résistance au cisaillement [3] d'un milieu poreux non saturé.

Les problèmes d'isolation hydrique, de géostructures énergétiques [4,5] ou encore de stockage de déchets radioactifs [6], font de la température une variable incontournable dans l'étude du comportement hydrique. En particulier, il est nécessaire de maîtriser l'influence de la température sur la courbe de rétention. Cet article présente une modélisation théorique et une étude expérimentale de cette influence dans le cas de matériaux poreux non saturés. A partir de la courbe de rétention d'eau obtenue expérimentalement à une température donnée, la modélisation permet de prédire cette courbe pour d'autres températures. On réduit ainsi considérablement le nombre d'essais expérimentaux nécessaires pour obtenir la courbe de rétention d'un milieu poreux à plusieurs températures.

La pertinence de cette modélisation est validée expérimentalement dans le cas d'une céramique en terre cuite et d'un sable limoneux argileux. L'application à d'autres résultats de la littérature $[7,8]$ montre sa capacité à couvrir une large gamme de matériaux.

\section{Modèle de comportement thermo-hydrique}

L'influence de la température sur le comportement hydrique d'un matériau poreux est due, en particulier, à la dilatation thermique des phases ainsi qu'aux variations de la tension superficielle du liquide et de l'angle de mouillage [9]. Le modèle proposé résulte du développement de la différentielle de la succion $s$ par rapport aux trois variables indépendantes : teneur en eau volumique $\theta$, température $T$ et indice des vides $e$. Seul un chemin de chargement hydrique en drainage, à partir d'un état saturé en eau chimiquement pure, est considéré ici. Ceci exclue toute situation faisant intervenir le phénomène d'hystérésis. La différentielle de la succion s'exprime sous la forme :

$$
\mathrm{d} s=\left(\frac{\partial s}{\partial \theta}\right)_{T, e} \mathrm{~d} \theta+\left(\frac{\partial s}{\partial T}\right)_{\theta, e} \mathrm{~d} T+\left(\frac{\partial s}{\partial e}\right)_{T, \theta} \mathrm{d} e
$$

Cette relation est explicitée dans la suite en exprimant les différents termes de dérivées partielles à partir de relations physiques. La teneur en eau volumique $\theta$ peut être exprimée en fonction des masses volumiques spécifiques des phases solide $\rho_{s}^{*}$ et liquide $\rho_{e}^{*}$, de la teneur en eau massique $w$ et de l'indice des vides $e$ par la relation :

$$
\theta=\frac{\rho_{s}^{*} w}{\rho_{e}^{*}(1+e)}
$$

La variation infinitésimale $\mathrm{d} \theta$, déduite de la relation (2), s'exprime sous la forme :

$$
\mathrm{d} \theta=\frac{\rho_{s}^{*}}{\rho_{e}^{*}(1+e)} \mathrm{d} w+\frac{\rho_{s}^{*} w}{\rho_{e}^{*}(1+e)}\left(\beta_{e}-\beta_{s}\right) \mathrm{d} T-\frac{\rho_{s}^{*} w}{\rho_{e}^{*}(1+e)^{2}} \mathrm{~d} e
$$

où $\beta_{e}$ et $\beta_{s}$ sont les coefficients de dilatation thermique de l'eau et de la phase solide. L'Éq. (2) conduit également à la relation :

$$
\left(\frac{\partial s}{\partial \theta}\right)_{T, e}=\frac{\rho_{e}^{*}(1+e)}{\rho_{s}^{*}}\left(\frac{\partial s}{\partial w}\right)_{T, e}
$$

Dans le contexte des études sur la rétention d'eau dans un milieu poreux, il est classique d'utiliser la loi de Jurin qui relie la succion à la tension superficielle de l'eau $\sigma_{s}$, au rayon moyen des pores $r$ et à l'angle de mouillage $\phi$ :

$$
s=\frac{2 \sigma_{s} \cos \phi}{r}
$$

La dérivée partielle de la succion par rapport à la température, déduite de la loi de Jurin (5) est :

$$
\left(\frac{\partial s}{\partial T}\right)_{\theta, e}=\frac{s}{\sigma_{s}} \frac{\mathrm{d} \sigma_{s}}{\mathrm{~d} T}-s \tan \phi \frac{\mathrm{d} \phi}{\mathrm{d} T}
$$

La relation (6) prend en compte le fait que la tension superficielle et l'angle de mouillage dépendent uniquement de la température. En notant $F_{w}$ l'inverse de la pente de la courbe de rétention à température et indice des vides constants $\left(F_{w}=\left(\frac{\partial s}{\partial w}\right)_{T, e}\right)$, et en introduisant (3), (4) et (6) dans (1), on obtient une nouvelle expression de la différentielle de la succion :

$$
\mathrm{d} s=F_{w} \mathrm{~d} w+\left(F_{w} w\left(\beta_{e}-\beta_{s}\right)+\frac{s}{\sigma_{s}} \frac{\mathrm{d} \sigma_{s}}{\mathrm{~d} T}-s \tan \phi \frac{\mathrm{d} \phi}{\mathrm{d} T}\right) \mathrm{d} T+\left(\left(\frac{\partial s}{\partial e}\right)_{T, \theta}-\frac{F_{w} w}{(1+e)}\right) \mathrm{d} e
$$


Le membre de droite de la relation (7) explicite trois termes qui traduisent les contributions respectives des variations de teneur en eau, de température et d'indice des vides, à la variation infinitésimale de la succion. Les deux premiers termes expriment un couplage fort entre la succion, la teneur en eau et la température. Le troisième terme devient négligeable devant les deux autres dans le cas d'un squelette solide peu déformable. Sa détermination expérimentale tout au long d'un cheminement thermohydromécanique n'est pas aisée. Par ailleurs, les variations infinitésimales de l'indice des vides s'entendent ici à contraintes mécaniques extérieures nulles, ce qui limite les déformations. Dans une première approche, ce terme sera négligé. La pertinence de ce choix sera discutée par la suite. De même, la variation de la masse volumique spécifique de la phase solide sera négligée devant celle de l'eau. Enfin, le manque de données expérimentales ne permet pas la prise en compte de la variation de l'angle de mouillage en fonction de la température. Toutes ces considérations conduisent à une expression simplifiée de la relation (7) sous la forme :

$$
\mathrm{d} s=F_{w} \mathrm{~d} w+\left(F_{w} w \beta_{e}+\frac{s}{\sigma_{s}} \frac{\mathrm{d} \sigma_{s}}{\mathrm{~d} T}\right) \mathrm{d} T
$$

A partir de la donnée de la courbe de rétention à la température $T_{0}$, la relation (8) permet de déterminer, pour chaque valeur de la succion, la variation de la teneur en eau en fonction de la variation de température. On obtient ainsi la courbe de rétention à une température quelconque $T$.

\section{Validation expérimentale}

\subsection{Dispositif expérimental et matériaux utilisés}

En vue de la validation du modèle, des essais expérimentaux sont réalisés au moyen d'un dispositif de type «plaque de pression » en température dont le schéma de principe est donné à la Fig. 1. La succion est appliquée par la méthode de surpression d'air [10]. L'échantillon se trouve dans une cellule hermétique dont la pression d'air est régulée par un circuit pneumatique. Il est disposé sur une céramique poreuse à haute pression d'entrée d'air permettant la continuité de la phase liquide entre l'échantillon et le circuit hydraulique. La cellule contenant l'échantillon est immergée dans un bain thermostaté, permettant ainsi de fixer la température de l'essai entre 20 et $70^{\circ} \mathrm{C}$. L'étude expérimentale de validation du modèle a porté sur deux matériaux :

- Le premier est une céramique en terre cuite. Sa masse volumique spécifique est $2738 \mathrm{~kg} \mathrm{~m}^{-3}$ et sa masse volumique apparente sèche $1870 \mathrm{~kg} \mathrm{~m}^{-3}$; son indice des vides est 0,464 . Avant tout chargement thermo-hydrique, les échantillons de céramique sont saturés en eau chimiquement pure; la teneur en eau massique de saturation est $16,9 \%$ à $20^{\circ} \mathrm{C}$.

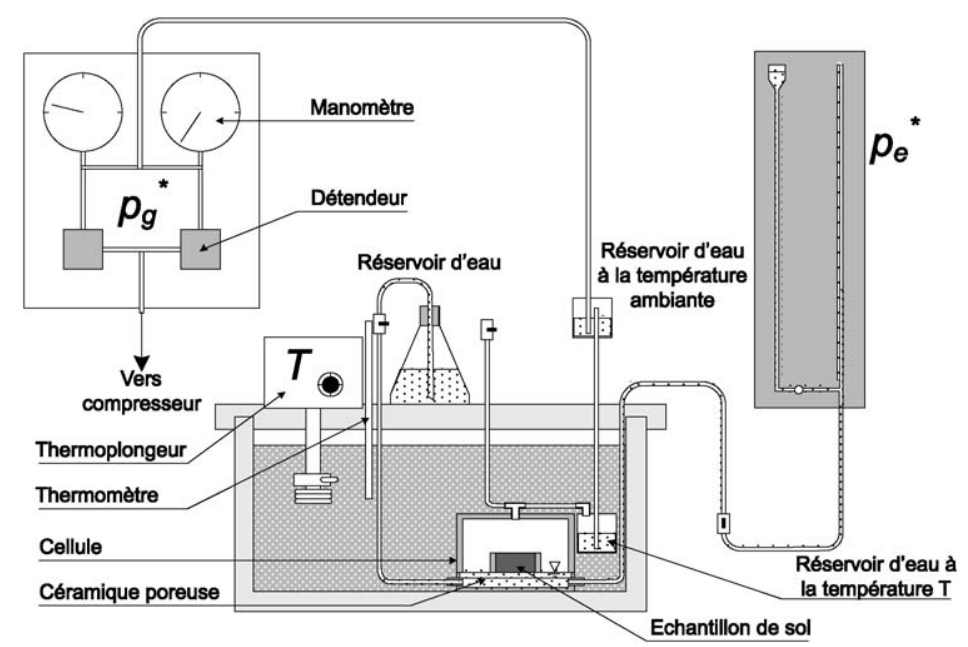

Fig. 1. Schéma de principe de la plaque de pression et de l'environnement de mesure.

Fig. 1. Pressure cell drawing in its environment. 

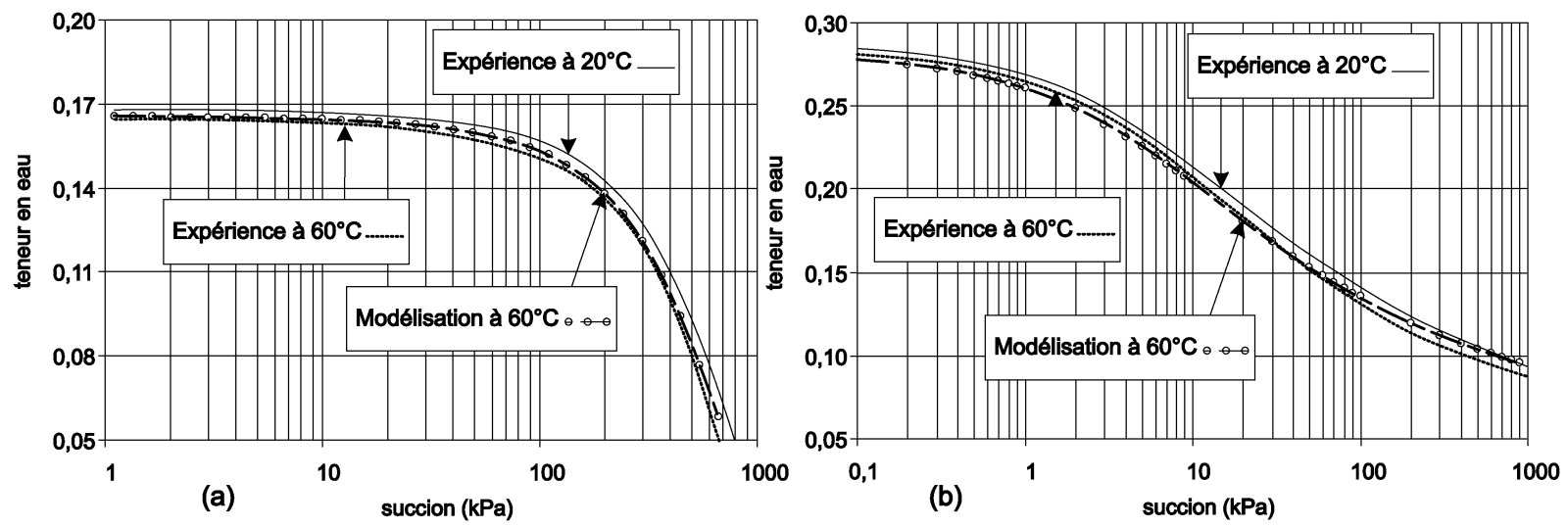

Fig. 2. Courbes de rétention; expérimentation et modélisation. Céramique (a) et sable limoneux argileux (b).

Fig. 2. Water retention curves; experiments and modelling. Ceramic (a) and clayey silty sand (b).

- Le deuxième matériau est un sable limoneux-argileux. Sa masse volumique spécifique est $2650 \mathrm{~kg} \mathrm{~m}^{-3}$. Les échantillons sont compactés pour atteindre une masse volumique apparente sèche de $1500 \mathrm{~kg} \mathrm{~m}^{-3}$, ce qui correspond à un indice des vides de 0,767 . Enfin, l'état initial saturé correspond à une teneur en eau massique de 28,9\% à $20^{\circ} \mathrm{C}$.

\subsection{Résultats expérimentaux et discussions}

La Fig. 2(a) montre la courbe de rétention de la céramique obtenue expérimentalement à 20 et $60^{\circ} \mathrm{C}$. La Fig. 2(b) donne les mêmes résultats pour le sable limoneux-argileux. Un lissage des points expérimentaux est réalisé en utilisant la fonction proposée par Fredlund et Xing [11]. La courbe de rétention à $20^{\circ} \mathrm{C}$ est utilisée comme courbe de référence pour le calcul décrit dans la Section 2. Le résultat de la modélisation, pour une température de $60^{\circ} \mathrm{C}$, est également montré sur la Fig. 2(a) pour la céramique et la Fig. 2(b) pour le sable limoneux-argileux. Les courbes de rétention issues de l'expérimentation et de la modélisation sont très proches dans les deux cas. En ce qui concerne le sable limoneux-argileux, on note une divergence à partir d'une succion qui correspond au passage du domaine funiculaire au domaine pendulaire dans lequel la continuité de la phase liquide n'est plus assurée.

Par la suite, le modèle présenté a été appliqué à d'autres résultats issus de la littérature. Il s'agit de mesures expérimentales réalisées sur deux argiles :

- La première est une argile compactée FoCa [7] composée d'environ 50\% de montmorillonite et de 50\% de kaolinite. Sa limite de liquidité est $112 \%$, sa limite de plasticité $50 \%$ et sa masse volumique spécifique $2675 \mathrm{~kg} \mathrm{~m}^{-3}$.

- La seconde est une argile remaniée, préparée à base d'argile de Boom [8]. Il s'agit d'une argile faiblement gonflante de composition 20-30\% kaolinite, 20-30\% illite, 10-20\% smectite. Sa limite de liquidité est 56\%, sa limite de plasticité $29 \%$ et sa masse volumique spécifique $2700 \mathrm{~kg} \mathrm{~m}^{-3}$.

La Fig. 3(a) montre les résultats obtenus pour l'argile compactée FoCa; les températures des essais sont 20 et $80^{\circ} \mathrm{C}$. On peut noter sur cette figure que la courbe de rétention, modélisée pour une température de $80^{\circ} \mathrm{C}$, est très proche de la courbe de rétention expérimentale obtenue à la même température.

La Fig. 3(b) présente les résultats de Romero [8] sur l'argile de Boom. Les essais de Romero ont été réalisés pour deux températures $\left(22\right.$ et $\left.80^{\circ} \mathrm{C}\right)$ et un poids volumique apparent sec de $16,7 \mathrm{kN} \mathrm{m}^{-3}$. Dans ce cas, le modèle présenté donne des prédictions pour $T=80^{\circ} \mathrm{C}$ en bon accord avec les résultats expérimentaux sur l'ensemble du domaine de variation de la succion. D'autres résultats obtenus par Romero [8], dans les mêmes conditions mais avec un poids volumique apparent sec de $13,7 \mathrm{kN} \mathrm{m}^{-3}$, sont également bien prédits par le modèle.

Les comparaisons entre modélisation et expérimentation, pour quatre matériaux différents, montrent la capacité de la relation (8) à prédire la courbe de rétention à n'importe quelle température dans la mesure où on dispose de la courbe de rétention à une température donnée $T_{0}$. L'hypothèse qui a conduit à négliger le troisième terme de la relation (7) devant les deux autres apparaît acceptable. En fait, bien que la variation de l'indice des vides puisse 

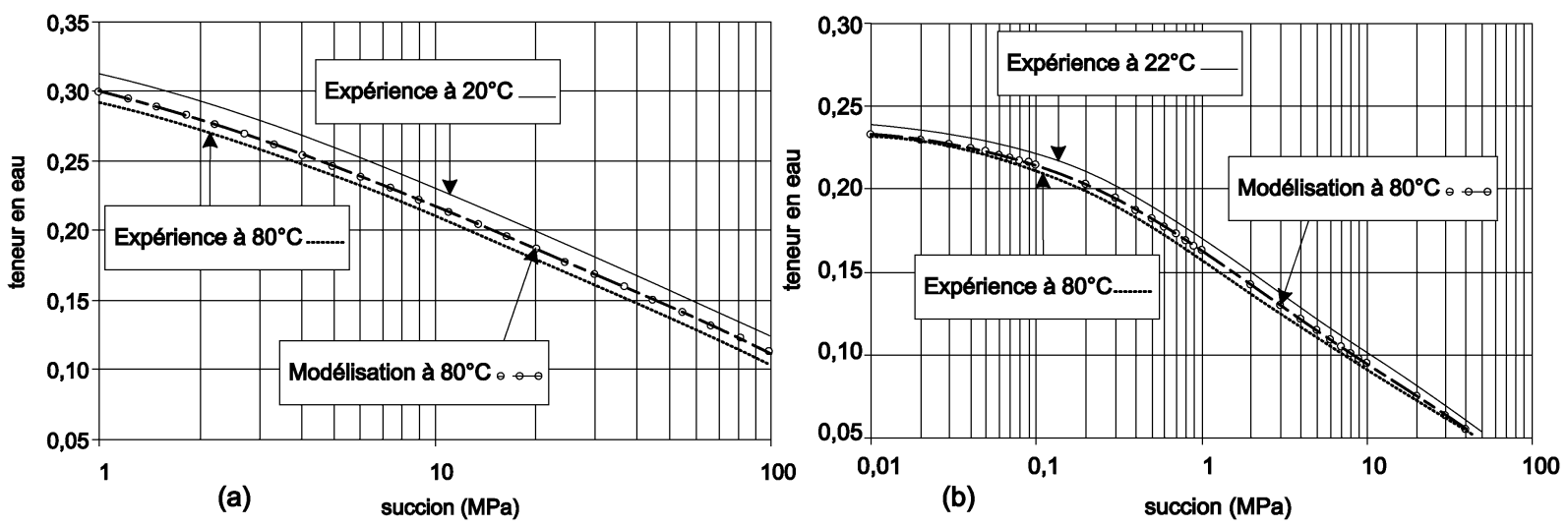

Fig. 3. Courbes de rétention; expérimentation et modélisation. Argile compactée FoCa [7] (a) et argile de Boom [8] (b).

Fig. 3. Water retention curves; experiments and modelling. Compacted clay FoCa [7] (a) and Boom clay [8] (b).

être non négligeable sur l'ensemble d'un essai, il ne semble pas nécessaire de prendre en compte explicitement cette variation dans le calcul. En effet, la détermination de la pente $F_{w}$ de la courbe de rétention intègre implicitement la contribution de la déformation progressive du matériau au cours du chargement hydrique.

Les résultats présentés dans cette note permettent d'envisager la généralisation de la courbe de rétention classique à une surface de rétention exprimant la teneur en eau en fonction de la succion et de la température.

\section{Conclusion}

Un modèle exprimant les variations de la succion en fonction des variables teneur en eau, température et indice des vides, a été proposé. Ce modèle, associé à la donnée d'une courbe de rétention à une température de référence, permet d'accéder à la courbe de rétention pour d'autres températures. Des expérimentations menées par les auteurs sur deux matériaux ainsi que des résultats empruntés à la littérature sur deux autres matériaux, ont permis de valider ce modèle.

Ces résultats permettent de réduire considérablement le nombre d'expérimentations dans le domaine des sciences des sols ou encore de l'exploitation géothermique des sous sols.

\section{Références}

[1] D.G. Fredlund, A. Xing, S. Huang, Predicting the permeability function for unsaturated soils using the soil-water characteristic curve, Can. Geotech. J. 31 (1994) 533-545.

[2] M.T. Van Genuchten, A closed-form equation for predicting the hydraulic conductivity of unsaturated soils, Soil Sci. Soc. Am. J. 44 (1980) 892-898.

[3] D.G. Fredlund, A. Xing, M.D. Fredlund, S.L. Barbour, The relationship of the unsaturated soil shear strength to the soil-water characteristic curve, Can. Geotech. J. 32 (1995) 440-448.

[4] C. Saix, Contribution à l'étude des sols non saturés en température. Application à l'exploitation thermique des sous-sols, Thèse de doctorat d'état, Université Montpellier II, 1987.

[5] L. Laloui, M. Moreni, L. Vulliet, Comportement d'un pieu bi-fonction, fondation et échangeur de chaleur, Can. Geotech. J. 40 (2003) $388-402$.

[6] E. Olchitzky, Couplage hydromécanique et perméabilité d'une argile gonflante non saturée sous sollicitations hydriques et thermiques, Thèse de doctorat, École Nationale des Ponts et Chaussées, 2002.

[7] C. Imbert, E. Olchitzky, T. Lassabatère, P. Dangla, A. Courtois, Evaluation of a thermal criterion for an engineered barrier system, Eng. Geol. 81 (2005) 269-283.

[8] E. Romero, A. Gens, A. Lloret, Temperature effects on the hydraulic behaviour of an unsaturated clay, Geotech. Geol. Eng. 19 (2001) $311-332$.

[9] J. Bachmann, R.R. Van Der Ploeg, A review on recent developments in soil water retention theory: interfacial tension and temperature effects, Soil Sci. 165 (2002) 468-478.

[10] F. Jamin, Contribution à l'étude du transport de matière et de la rhéologie dans les sols non saturés à différentes températures, Thèse de doctorat, Université Montpellier II, 2003.

[11] D.G. Fredlund, A. Xing, Equations for the soil-water characteristic curve, Can. Geotech. J. 31 (1994) 521-532. 